\title{
Improvement of Diagnostic Accuracy for Pancreatic Cancer with Serum Lactate Dehydrogenase
}

\author{
Xi Wang ${ }^{1, *}$ \\ Chunbin Wang ${ }^{2, *}$ \\ Haijun Zhang (D) \\ 'Department of Oncology, Zhongda \\ Hospital, Medical School of Southeast \\ University, Nanjing, Jiangsu Province, \\ People's Republic of China; ${ }^{2}$ Department \\ of Oncology, Yancheng Third People's \\ Hospital, The Affiliated Yancheng \\ Hospital of Southeast University Medical \\ College, Yancheng, Jiangsu Province, \\ People's Republic of China \\ *These authors contributed equally to \\ this work
}

Correspondence: Haijun Zhang Department of Oncology, Zhongda Hospital, Medical School of Southeast University, 87 Dingjiaqiao Road, Nanjing, 210009, Jiangsu Province, People's

Republic of China

$\mathrm{Tel} / \mathrm{Fax}+862583272216$

Email zhanghaijunseu@I63.com
Purpose: Due to the lack of early-stage detection, pancreatic cancer (PC) remains a devastating disease worldwide. Lactate dehydrogenase (LDH) is associated with tumorigenesis and cancer progression. This study aims to analyze the diagnostic improvements in serum LDH levels combined with other common tumor biomarkers, including carbohydrate antigen 19-9 (CA19-9) and carcinoembryonic antigen (CEA), for monitoring PC.

Patients and Methods: A retrospective analysis was performed on 73 patients with newly diagnosed PC, 90 patients with pancreatic benign diseases (PBD), and 92 people with healthy physical examination (HPE) at Zhongda Hospital, Southeast University from July 2013 to July 2020. The diagnostic efficiencies of serum levels of LDH, CA19-9, and CEA were analyzed through receiver operating characteristic (ROC) curves for PC. The sensitivity and specificity were evaluated at an optimal cutoff. The prognostic impacts of LDH on PC patients were also assessed.

Results: The LDH level was elevated in 21 (28.77\%) patients with PC, 3 (3.33\%) PBD patients, and no HPE individuals $(P<0.05)$. The sensitivities of LDH, CA19-9, and CEA for the diagnosis of $\mathrm{PC}$ were $63.0 \%, 78.1 \%$, and $72.6 \%$, respectively, but the combination of these three markers increased predictive sensitivity significantly to $87.6 \%$. The specificities of LDH, CA19-9, and CEA for the diagnosis of PC were $93.4 \%, 84.1 \%$, and $73.1 \%$, respectively. The combined specificity reached up to $96.7 \%$. The medium survival time of PC patients with low-level LDH was $21 \pm 5.1$ months, whereas that of patients with highlevel LDH was only $7 \pm 0.92$ months $(P<0.05)$.

Conclusion: The serum LDH level was higher in PC patients than in PBD patients and HPE individuals and was associated with a poor prognosis. The combined assessment of LDH, CEA, and CA19-9 showed higher sensitivity and specificity for the diagnosis of PC.

Keywords: pancreatic cancer, lactate dehydrogenase, diagnosis

\section{Introduction}

Pancreatic cancer (PC) is among the worst of all cancer maladies, as the tenth most commonly diagnosed cancer in the USA and the fourth most common cause of all cancer-related deaths. ${ }^{1,2}$ The 5-year survival rate of PC patients is the lowest for all cancer types, at approximately $9 \% .{ }^{1}$ At the time of diagnosis, nearly $80 \%$ of PC patients have already reached an advanced disease stage, which is often past the window for treatment efficacy, resulting in a high mortality rate. ${ }^{3}$ The lack of accurate detection tests capable of identifying early-stage PC is a primary underlying contributor to poor prognosis in patients with PC.

Currently, the diagnosis of PC is hugely dependent on the detection of tumor biomarkers and imaging features. Carbohydrate antigen 19-9 (CA19-9) and 
carcinoembryonic antigen (CEA) are common biomarkers for PC. Although CA19-9 has satisfactory specificity for differentiating benign from malignant pancreatic tumors, CA 19-9 has poor sensitivity ${ }^{4}$ and can also be elevated in hepatobiliary cancers and benign biliary obstruction. ${ }^{5}$ Furthermore, imaging is an important component of PC diagnosis; ${ }^{6}$ however, each imaging modality has different advantages and disadvantages, which can require costly equipment and specialists. Histological diagnosis often requires invasive operations, such as biopsy obtained by endoscopic ultrasonography-guided fine-needle aspiration (EUS-FNA). Therefore, the accurate diagnosis of PC patients based on radiological features and CA19-9 has thus far been unsatisfactory.

Compared with normal cells, cancer cells will switch from oxidative phosphorylation to increased glycolysis in the absence of sufficient oxygen, ${ }^{7}$ which is also called the "Warburg effect." Lactate dehydrogenase (LDH) is a central player in the Warburg effect, and elevated LDH levels in many cancer cell types have been associated with the growth, maintenance, and invasion of cancer cells, as demonstrated in Figure 1. Many studies have indicated the importance of this potential biomarker in cancer diagnosis and prediction and have suggested $\mathrm{LDH}$ as a potential therapeutic target in cancer. ${ }^{8-10}$ As a prognostic biomarker, LDH is elevated in multiple cancers and has been associated with poor survival. ${ }^{11-17}$

The combination of CA19-9 with either CEA or carbohydrate antigen 125 (CA125) can improve the specificity and sensitivity of $\mathrm{PC}$ diagnosis for $\mathrm{PC}$, respectively. ${ }^{18}$ However, whether analyzing each test individually or analyzing the tests in combination leads to better PC diagnostic outcomes remains unclear based on currently available studies. With the aim of improving the sensitivity and specificity of PC diagnosis, this study evaluated the accuracy of evaluating serum $\mathrm{LDH}$ levels together with the common tumor biomarkers CA19-9 and CEA.

\section{Patients and Methods}

\section{Study Population}

A retrospective study was performed, including consecutive patients with newly diagnosed PC or pancreatic benign diseases (PBD) who were admitted to Zhongda Hospital, Southeast University, in addition to healthy individuals recruited from the outpatient department, from July 2013 to July 2020. The inclusion criteria were as follows: 1) PC patients were confirmed by histopathological resection and fine-needle aspiration cytology, without receiving any chemotherapy, radiotherapy, molecular targeted therapy, or immunotherapy before surgery; 2) patients with newly diagnosed PBD, including benign pancreatic tumor, intraductal papillary mucinous neoplasm (IPMN), pancreatic pseudocyst, and chronic pancreatitis; and 3) healthy physical examination (HPE) individuals included all eligible volunteers without any pancreatic diseases or malignant tumors. The exclusion criterion was patients with a prior history of any other malignant tumor. All patients signed informed consent forms. The study was approved by the ethics committee of Zhongda Hospital, Southeast University and followed a study protocol that protected the private information of enrolled patients, in accordance with the provisions of the Helsinki Declaration.

\section{Measurement of Markers in Blood Samples}

A volume of $5 \mathrm{~mL}$ fasting peripheral venous blood was collected from each patient during the first 24 hours of admission, which was processed using routine centrifugation at $4{ }^{\circ} \mathrm{C}$ to separate the serum. $\mathrm{LDH}$ catalyzes the reaction: L-lactate + Nicotinamide adenine dinucleotide $(\mathrm{NAD}+)$ in equilibrium with pyruvate $+\mathrm{NADH}$. The reaction was monitored by measuring the increase in NADH at $340 \mathrm{~nm}$ using a Beckman Coulter AU680, according to the manufacturer's protocol. Serum LDH levels were calculated based on the rate method, using the International Federation of Clinical Chemistry-recommended procedure. In our hospital, the normal range for serum $\mathrm{LDH}$ is 120-250 U/L; thus, serum LDH level $>250 \mathrm{U} / \mathrm{L}$ is considered to be high. CA19-9 levels in serum were measured according to the principle of the double antibody sandwich method using an automatic electrochemiluminescence immunoassay system (purchased from Roche). The normal range for serum CA19-9 is 0-39 U/mL, such that serum CA19-9 level $>39 \mathrm{U} / \mathrm{L}$ is considered to be high. According to the manufacturer's protocol, the serum CEA level was detected using a chemiluminescent immunoassay (purchased from Roche), which has a normal reference value in the range of $0-5 \mu \mathrm{g} / \mathrm{L}$.

\section{Data Collection}

Basic data were collected, including demographic information (such as age and sex). Tumor characteristics, including primary tumor site, tumor size, tumor type, differentiation 


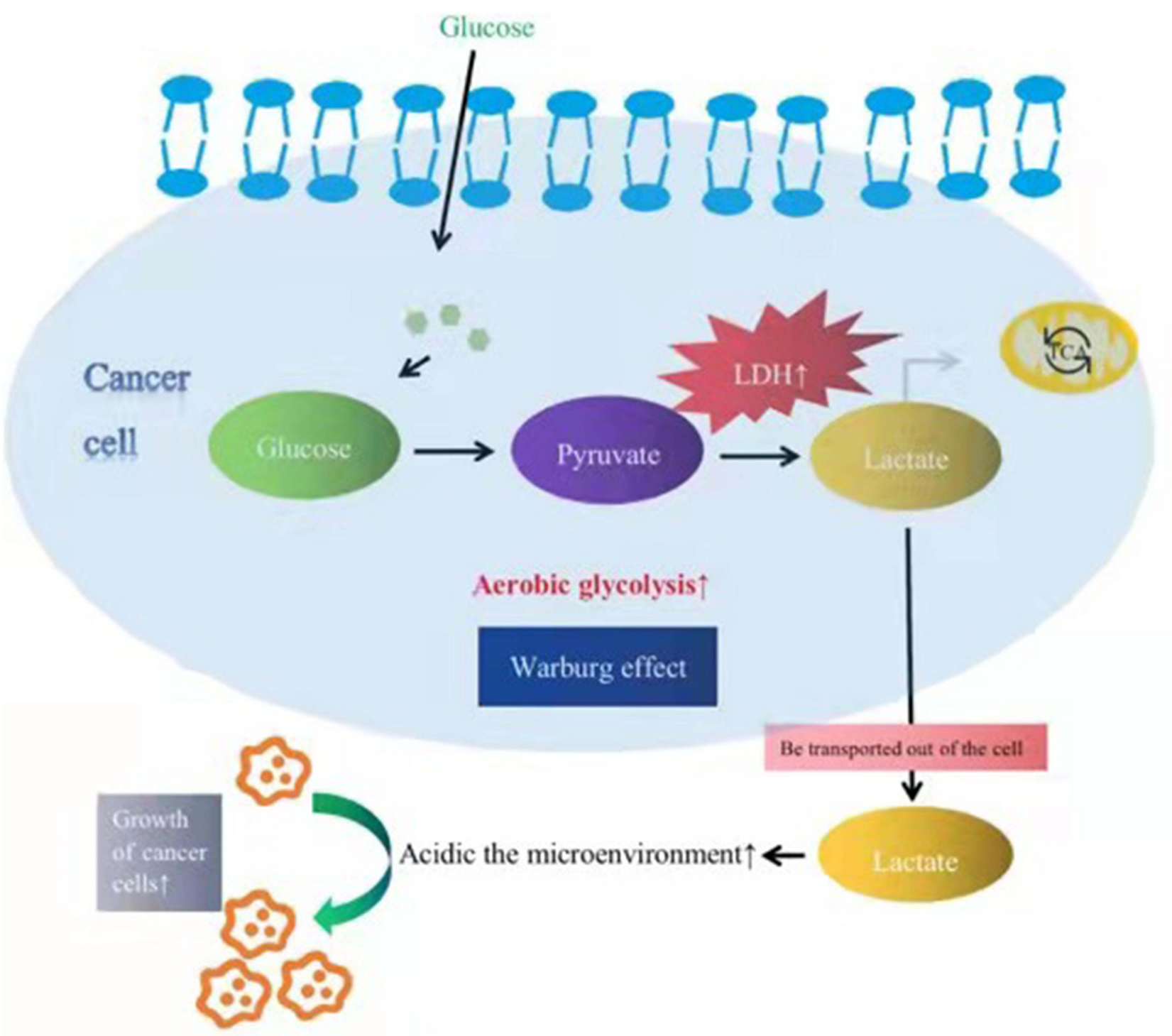

Figure I Scheme of the relationships among LDH, Warburg effect, and cancer cells. Abbreviation: LDH, lactate dehydrogenase.

stage (well, moderate, or poor), tumor (T) status, lymph node $(\mathrm{N})$ status, and tumor, node, metastasis (TNM) stages were reviewed and analyzed according to the detailed descriptions on each patient's pathology report.

\section{Statistical Analysis}

Statistical analysis was conducted using SPSS 25.0 software. The normally distributed data were analyzed using t-tests, and the categorical data were analyzed using chisquare tests. The mean levels of biomarkers were compared using analysis of variance (ANOVA). The discriminatory abilities of LDH, CA19-9, CEA, and various combinations of these factors were assessed using receiver operating characteristic (ROC) curves. In addition, the area under the curve (AUC), sensitivity, and specificity at an optimal cutoff value were evaluated after minimizing the total prediction error with MedCalc software. The survival time was estimated by Kaplan-Meier analysis. The survival differences between groups were compared using the Log rank test. $\mathrm{P}<0.05$ was considered significant.

\section{Results}

\section{Clinical Characteristics}

A total of 73 patients with newly diagnosed PC were enrolled as the experimental group in this study, and their tumor characteristics are provided in Table 1. 
Table I Tumor Characteristics of Pancreatic Cancer

\begin{tabular}{|c|c|c|}
\hline \multicolumn{2}{|c|}{ Tumor Characteristics } & \multirow{2}{*}{$\begin{array}{l}\text { Numbers } \\
40 \text { (54.8\%) }\end{array}$} \\
\hline Primary tumor site & Head or neck (\%) & \\
\hline & Body or tail (\%) & $31(42.5 \%)$ \\
\hline & Other (\%) & $2(2.7 \%)$ \\
\hline Tumor size & Mean $(\mathrm{cm})$ & $3.68 \pm 2.85$ \\
\hline \multirow[t]{2}{*}{ Tumor type } & $\begin{array}{l}\text { Ductal adenocarcinoma } \\
\qquad(\%)\end{array}$ & 65 (89.0\%) \\
\hline & Other (\%) & $8(11.0 \%)$ \\
\hline \multirow[t]{4}{*}{ Tumor status $(\mathrm{T})$} & I (\%) & $12(16.4 \%)$ \\
\hline & $2(\%)$ & 14 (19.2\%) \\
\hline & $3(\%)$ & 9 (12.3\%) \\
\hline & $4(\%)$ & $38(52.1 \%)$ \\
\hline \multirow{4}{*}{$\begin{array}{l}\text { Lymph node status } \\
\text { (N) }\end{array}$} & $0(\%)$ & $33(45.2 \%)$ \\
\hline & I (\%) & 31 (42.5\%) \\
\hline & $2(\%)$ & 7 (9.6\%) \\
\hline & Not clear (\%) & $2(2.7 \%)$ \\
\hline \multirow[t]{2}{*}{ Metastasis (M) } & 0 (\%) & $36(49.3 \%)$ \\
\hline & I (\%) & 37 (50.7\%) \\
\hline \multirow[t]{4}{*}{ Tumor stage } & I (\%) & $16(21.9 \%)$ \\
\hline & II (\%) & $12(16.4 \%)$ \\
\hline & III (\%) & $8(11.0 \%)$ \\
\hline & IV (\%) & 37 (50.7\%) \\
\hline \multirow[t]{3}{*}{ Differentiation } & Poor (\%) & $20(27.4 \%)$ \\
\hline & Moderate (\%) & 49 (67.1\%) \\
\hline & Well (\%) & $4(5.5 \%)$ \\
\hline
\end{tabular}

Additionally, 90 patients with newly diagnosed PBD and 92 HPE individuals were selected as control groups. Among patients with newly diagnosed PBD, 15 cases were benign pancreatic tumors, 56 were IPMN, 10 were pancreatic pseudocysts, and 9 were chronic pancreatitis. The demographic information for the experimental and control groups is presented in Table 2. The average ages of the PC, PBD, and HPE groups were $64.21 \pm 8.82,63.98$ \pm 15.06 , and $58.14 \pm 10.99$ years, respectively. No significant differences in age or sex were observed between groups. Information regarding the treatments administered to the PC patients is listed in Table 3. Interestingly, by performing qualitative analysis, relying on the normal reference value (120-250 U/L), $28.77 \%$ of patients with PC were preliminarily found to have elevated LDH levels (>250 U/L); however, only $3.33 \%$ of PBD patients and none of the HPE individuals had elevated LDH levels, indicating that the frequency of elevated LDH was higher among PC patients than PBD patients and HPE individuals $(P<0.05)$. Unsurprisingly, compared with HPE individuals, more PC patients and PBD patients presented with elevated CA19-9 and CEA levels, especially PC patients.

\section{Comparisons of LDH, CA19-9, and CEA Levels in Patients}

Furthermore, the levels of LDH, CA19-9, and CEA were compared through quantitative analysis in patients with $\mathrm{PC}$, patients with PBD, and HPE people. As listed in Table 4, the serum LDH level in PC patients was higher than those in both PBD patients and HPE individuals $(P<0.05)$. The results also demonstrated that serum levels of CA19-9 were higher in both PC patients and PBD patients than in HPE individuals $(P<0.05)$, and the level of CA19-9 in PC patients was higher than that in PBD patients $(P<0.05)$. Similar results were observed for CEA levels. Among the three groups, the CEA level significantly increased in the ascending order of HPE individuals, PBD patients, and PC patients $(P<0.05)$. Moreover, Figure 2 shows that the LDH levels in PC patients at Stage III/IV were significantly higher than those of patients at Stage I/II $(P=0.002)$.

\section{Diagnostic Accuracy for PC When Using Individual Markers and Combinations of LDH, CA 19-9, and CEA}

To investigate the diagnostic accuracy of serum LDH for PC, we generated an ROC curve, as shown in Figure 3, and determined the diagnostic efficiency, shown in Table 5, of using individual markers and combinations of predictors. The individual AUC values for LDH, CA19-9, and CEA were $0.815 \pm 0.036,0.821 \pm 0.036$, and $0.784 \pm$ 0.035 , respectively. When LDH was combined with the common tumor biomarkers CA19-9 and CEA, the AUC reached as high as $0.874 \pm 0.029$. The thresholds established for LDH, CA19-9, and CEA for the diagnosis of PC were $209 \mathrm{U} / \mathrm{L}, 29.63 \mathrm{U} / \mathrm{L}$, and $2.99 \mathrm{ng} / \mathrm{mL}$, respectively. The sensitivities of LDH, CA19-9, and CEA for the diagnosis of PC were $63.0 \%, 78.1 \%$, and $72.6 \%$, respectively; however, a noteworthy increase was observed when using 
Table 2 Baseline Characteristics of Experimental Group and Control Groups

\begin{tabular}{|c|c|c|c|c|c|}
\hline \multicolumn{2}{|l|}{ Characteristics } & \multirow{3}{*}{$\begin{array}{c}\text { Experimental Group } \\
\text { PC (N=73) } \\
52(71.23 \%)\end{array}$} & \multicolumn{2}{|c|}{ Control Group } & \multirow[t]{2}{*}{$p$} \\
\hline & & & PBD $(\mathrm{N}=90)$ & HPE $(\mathrm{N}=92)$ & \\
\hline \multirow[t]{2}{*}{ Gender } & Male (\%) & & $60(66.67 \%)$ & 66 (7I.74\%) & \multirow[t]{2}{*}{0.759} \\
\hline & Female (\%) & 21 (28.77\%) & $30(33.33 \%)$ & $26(28.26 \%)$ & \\
\hline Age (year) & Mean & $64.21 \pm 8.82$ & $63.98 \pm 15.06$ & $58.14 \pm 10.99$ & 0.903 \\
\hline \multirow[t]{2}{*}{ LDH (U/L) } & $\leq 250$ & 52 (7I.23\%) & 87 (96.67\%) & $92(100 \%)$ & \multirow[t]{2}{*}{$0.000 *$} \\
\hline & $>250$ & $2 \mathrm{I}$ (28.77\%) & $3(3.33 \%)$ & $0(0 \%)$ & \\
\hline \multirow[t]{2}{*}{ CEA (ng/L) } & $\leq 5$ & 37 (50.69\%) & 81 (90.00\%) & 87 (94.57\%) & \multirow[t]{2}{*}{$0.000 *$} \\
\hline & $>5$ & 36 (49.3।\%) & $9(10.00 \%)$ & 5 (5.43\%) & \\
\hline \multirow[t]{2}{*}{ CAI9-9 (U/mL) } & $\leq 39$ & $20(27.40 \%)$ & 70 (77.78\%) & 86 (93.48\%) & \multirow[t]{2}{*}{$0.000^{*}$} \\
\hline & $>39$ & $53(72.60 \%)$ & $20(22.22 \%)$ & $6(6.52 \%)$ & \\
\hline
\end{tabular}

Note: $*<0.05$.

Abbreviations: PC, pancreatic cancer; PBD, pancreatic benign disease; HPE, healthy physical examination; LDH, lactate dehydrogenase; CAI9-9, carbohydrate antigen I99; CEA, carcino-embryonic antigen.

the combined predictors, with a sensitivity of $87.6 \%$. The specificities of LDH, CA19-9, and CEA for the diagnosis of PC were $93.4 \%, 84.1 \%$, and $73.1 \%$, respectively. When LDH was combined with CA19-9 and CEA, the specificity for PC diagnosis reached as high as $96.7 \%$.

\section{Overall Survival of PC Patients with Low or High Levels of LDH}

PC patients were divided into two groups based on the defined threshold (209 U/L) for LDH. Information regarding the treatment of PC patients is shown in Table 3. As shown in Figure 4, the medium survival time of PC patients with low LDH levels (below the threshold value of $209 \mathrm{U} / \mathrm{L}$ ) was $21 \pm$ 5.1 months, whereas that for patients with high LDH levels was only $7 \pm 0.92$ months $(P<0.05)$. High levels of LDH in PC patients significantly contributed to poor prognosis.

Table 3 Treatments for Patients with Pancreatic Cancer

\begin{tabular}{|l|c|}
\hline Treatment & Numbers (\%) \\
\hline No special treatment & $10(13.70 \%)$ \\
\hline Surgery & $25(34.25 \%)$ \\
\hline Intravenous chemotherapy & $15(20.55 \%)$ \\
\hline Surgery+chemotherapy & $10(13.70 \%)$ \\
\hline Radioactive iodide placement & $3(4.11 \%)$ \\
\hline Others & $10(13.70 \%)$ \\
\hline
\end{tabular}

\section{Discussion}

Our findings in this study revealed that the serum LDH levels of PC patients were higher than those of patients with PBD and HPE individuals. A significant association was also found among LDH levels, tumor stages, and poor prognosis. When LDH was combined with the common tumor biomarkers CA19-9 and CEA, the sensitivity and specificity of PC diagnosis significantly increased, improving diagnostic accuracy.

The low 5-year survival rate of PC highlights the necessity of developing more accurate diagnostic techniques. ${ }^{19}$ CA19-9 is the only marker that has been approved by the United States Food and Drug Administration for use in the routine management of PC. ${ }^{20}$ CEA can supplement CA19-9 with a specificity of $84 \%{ }^{21} \mathrm{CA} 125$ has encouraging sensitivity and specificity for the diagnosis of PC when combined with CA19-9. ${ }^{22}$ Studies have reported that members of the mucin family, such as MUC1 and MUC4, glypican, fibroblasts, complement $\mathrm{C} 3$, complement $\mathrm{C} 4$, and apolipoprotein, could be used as indicators for PC diagnosis, prognosis prediction, and recurrence monitoring. ${ }^{23}$ However, most of these remain in the experimental stage due to the complexity of detection or the lack of internationally standardized cutoff points. ${ }^{24}$ Imaging modalities used in PC diagnosis may fail to detect early lesions or be unable to distinguish between benign and malignant lesions. ${ }^{25}$ However, the analysis of tumor-associated markers in blood cells is a simple and easy technique that can be performed at most hospitals; therefore, identifying cheaper 
Table 4 Serum Level of LDH, CAI9-9, and CEA in PC Patients, PBD Patients and HPE People

\begin{tabular}{|l|c|c|c|}
\hline Groups & LDH (U/L) & CA19-9 (U/mL) & CEA (ng/L) \\
\hline PC & $218(184-270)$ & $156.50(35.12-913.85)$ & $2.96(2.52-12.25)$ \\
\hline PBD & $179(155-200)$ & $13.38(7.09-30.96)$ & $1.91(1.18-2.69)$ \\
\hline HPE & $178(160-191)$ & $8.99(5.12-14.18)$ & 0.000 \\
\hline$P$ & 0.000 & 0.000 & $<0.050$ \\
\hline$P_{1}$ & $<0.050$ & $<0.050$ & $<0.050$ \\
\hline$P_{2}$ & $<0.050$ & $<0.050$ & $<0.050$ \\
\hline$P_{3}$ & 0.687 & $<0.050$ & \\
\hline
\end{tabular}

Note: $P_{1}, P C$ vs PBD; $P_{2}$, PC vs HPE; $P_{3}$, PBD vs HPE.

Abbreviations: PC, pancreatic cancer; PBD, pancreatic benign disease; HPE, healthy physical examination; LDH, lactate dehydrogenase; CAI9-9, carbohydrate antigen 199; CEA, carcino-embryonic antigen.

and more accurate serum biomarkers can improve the current unsatisfactory PC diagnostic outcomes.

LDH is a tetrameric enzyme comprised of two types of subunits: LDHA (muscle-type, $\mathrm{M}$ subunit) and LDHB (heart-type, $\mathrm{H}$ subunit). ${ }^{23}$ LDHA is the primary form found in skeletal muscle, which converts pyruvate to lactic acid. According to the Warburg effect, glycolysis is more active in tumor cells than in normal cells. The level of LDHA is higher in human tumor cells than in normal tissue, ${ }^{26}$ and the knockdown of the LDH gene can attenuate glycolysis and inhibit tumor cell progression. ${ }^{27} \mathrm{LDH}$ has been suggested as a potential diagnostic biomarker for malignant tumors. In breast cancer, gynecological cancer, non-Hodgkin lymphoma, lung cancer, and esophageal squamous cell cancer patients, higher levels of serum LDH have been detected..$^{8-11}$

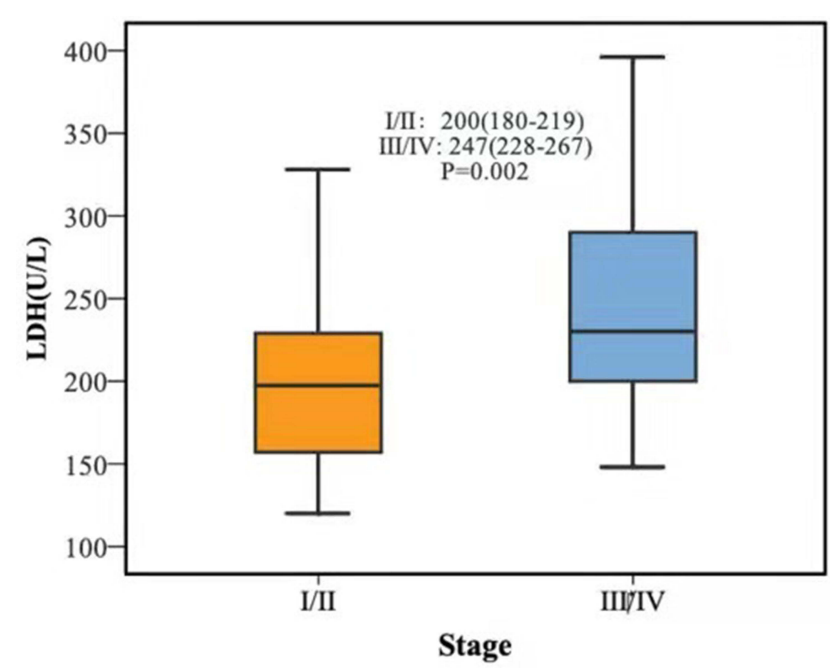

Figure 2 Levels of LDH in PC patients with Stage I/II or Stage III/IV disease. Abbreviations: LDH, lactate dehydrogenase; PC, pancreatic cancer.
LDHA overexpression was detected throughout the tumor process in nearly all cell lines tested. High levels of LDHA may have a marked adverse influence on patient survival. In addition, the activity of alcohol dehydrogenase (ADH) class III isoenzymes in cancerous tissues is approximately and significantly $30 \%$ higher than that in healthy tissues. ${ }^{28}$ Jelski et al reported that the expression of class III ADH isoenzymes was reflected in the serum of patients with $\mathrm{PC},{ }^{29,30}$ whereas other types of ADH isoenzymes (I, II, IV) showed no significant change in either pancreatic tissue or serum. The release of class III ADH isoenzymes from cancer cells causes the total ADH activity to significantly increase. Our results revealed that in the serum of PC patients, which is easy to obtain, the level of LDH was higher than those in both

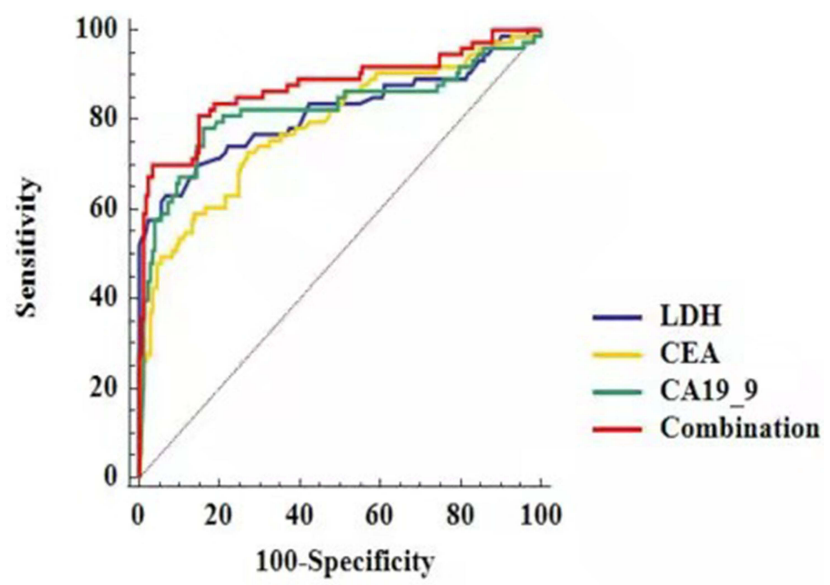

Figure 3 ROC for the diagnostic efficiency of serum LDH, CEA, and CAI9-9, both individually and combined, for PC testing.

Abbreviations: ROC, receiver operating characteristic curves; $L D H$, lactate dehydrogenase; CA19-9, carbohydrate antigen 19-9; CEA, carcinoembryonic antigen; PC, pancreatic cancer. 
Table 5 Diagnostic Accuracy of Individual Markers and Combined Predictors of LDH, CAI9-9, and CEA for PC

\begin{tabular}{|c|c|c|c|c|c|c|c|c|c|}
\hline \multirow[t]{2}{*}{ Biomarker } & \multirow[t]{2}{*}{ AUC } & \multirow[t]{2}{*}{ Se } & \multirow[t]{2}{*}{$\mathbf{P}$} & \multicolumn{2}{|c|}{$95 \% \mathrm{Cl}$} & \multirow[t]{2}{*}{ Optimal Cutoff } & \multirow[t]{2}{*}{ Sensitivity\% } & \multirow[t]{2}{*}{ Specificity\% } & \multirow[t]{2}{*}{ Youden Index } \\
\hline & & & & Upper & Lower & & & & \\
\hline LDH & 0.815 & 0.036 & $<0.05$ & 0.762 & $0.86 I$ & $209 \mathrm{U} / \mathrm{L}$ & 63.0 & 93.4 & 0.564 \\
\hline CAI9-9 & 0.821 & 0.036 & $<0.05$ & 0.768 & 0.866 & $29.63 \mathrm{U} / \mathrm{L}$ & 78.1 & 84.1 & 0.622 \\
\hline CEA & 0.784 & 0.035 & $<0.05$ & 0.728 & 0.833 & $2.99 \mathrm{ng} / \mathrm{mL}$ & 72.6 & 73.1 & 0.457 \\
\hline Combinations & 0.874 & 0.029 & $<0.05$ & 0.826 & 0.912 & & 87.6 & 96.7 & 0.843 \\
\hline
\end{tabular}

Abbreviations: LDH, lactate dehydrogenase; CAI9-9, carbohydrate antigen 19-9; CEA, carcino-embryonic antigen; PC, pancreatic cancer; AUC, area under the curve; Cl, confidence interval.

HPE individuals and PBD patients, which is consistent with the findings reported for other cancers. When LDH was combined with the common biomarkers CA19-9 and CEA, the specificity for PC diagnosis significantly increased to $96.7 \%$, and the sensitivity increased to $87.6 \%$. Furthermore, our results demonstrated that serum LDH levels could act as a useful predictor of estimated survival.

Our study has some limitations. First, the number of PC patients was small. Second, we did not compare the levels of LDH in cancer tissues and with that in normal healthy tissue.

The relationship between cancerous cells and the release of serum LDH has not been clarified clearly. Finally, the sensitivity of serum LDH was lower than that of the traditional marker (CA19-9), although the specificity of LDH was

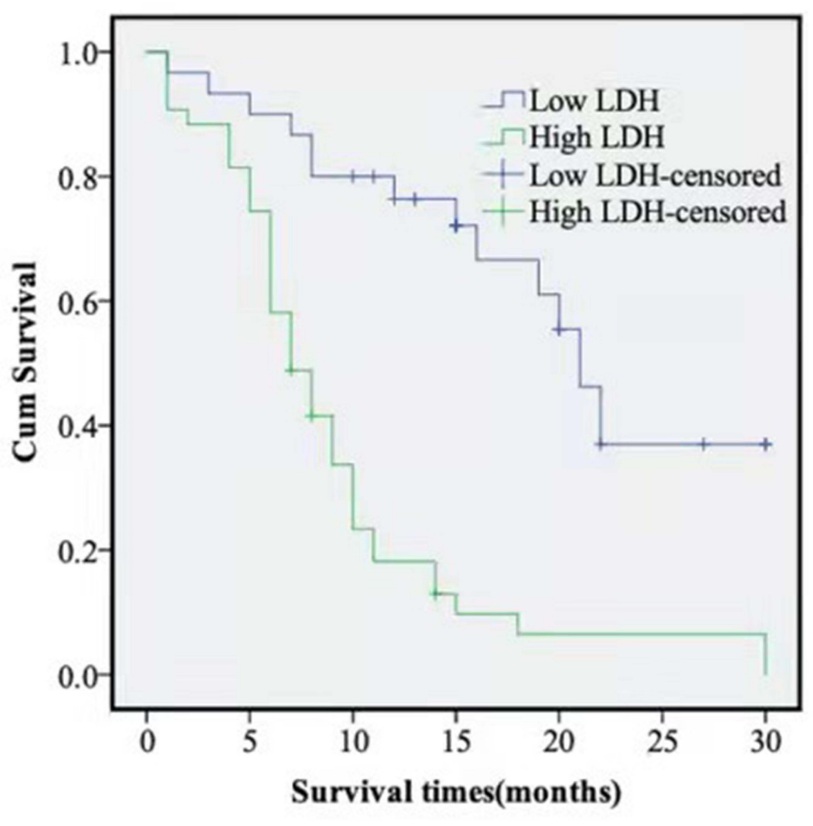

Figure 4 Effect of LDH on overall survival in patients with PC.

Abbreviations: Low LDH, low level of lactate dehydrogenase; High LDH, highlevel of lactate dehydrogenase; PC, pancreatic cancer. higher than those of CA19-9 and CEA. Additional prospective research in a larger patient sample remains necessary to improve the diagnostic accuracy of PC.

\section{Conclusion}

In conclusion, serum LDH levels were higher in PC patients than in PBD patients and HPE individuals. The test combining LDH, CEA, and CA19-9 had higher sensitivity and specificity, which may improve the diagnosis of PC.

\section{Disclosure}

The authors report no conflicts of interest in this work.

\section{References}

1. Siegel R, Ma J, Zou Z, et al. Cancer statistics, 2019. CA Cancer J Clin. 2019;64:9-29. doi:10.3322/caac.21208

2. Rahib L, Smith BD, Aizenberg R, et al. Projecting cancer incidence and deaths to 2030: the unexpected burden of thyroid, liver, and pancreas cancers in the United States. Cancer Res. 2014;74:2913-2921. doi:10.1158/0008-5472.CAN-14-0155

3. Wu H, Chen X, Ji J, et al. Progress of exosomes in the diagnosis and treatment of pancreatic cancer. Genet Test Mol Biomarkers. 2019;23 (3):215-222. doi:10.1089/gtmb.2018.0235

4. Cao S, Hu Y, Gao X, et al. Serum carbohydrate antigen 19-9 in differential diagnosis of benign and malignant pancreatic cystic neoplasms: a meta-analysis. PLoS One. 2016;11(11):e0166406. doi:10.1371/journal.pone. 0166406

5. Winter JM, Cameron JL, Campbell KA, et al. 1423 pancreaticoduodenectomies for pancreatic cancer: a single-institution experience. J Gastrointest Surg. 2006;10(9):1199-1210. doi:10.1016/j.gassur.2006.08.018

6. Lee ES, Lee JM. Imaging diagnosis of pancreatic cancer: a state-of-the -art review. World J Gastroenterol. 2014;20(24):7864-7877. doi:10.3748/wjg.v20.i24.7864

7. Vander Heiden MG, Cantley LC, Thompson CB. Understanding the Warburg effect: the metabolic requirements of cell proliferation. Science. 2009;324(5930):1029-1033. doi:10.1126/science.1160809

8. Koukourakis MI, Kontomanolis E, Giatromanolaki A, et al. Serum and tissue LDH levels in patients with breast/gynaecological cancer and benign diseases. Gynecol Obstet Invest. 2009;67(3):162-168. doi: $10.1159 / 000183250$

9. Benboubker L, Valat C, Linassier C, et al. A new serologic index for low-grade non-Hodgkin's lymphoma based on initial CA125 and LDH serum levels. Ann Oncol. 2000;11:1485-1491. doi:10.1023/ A: 1026789232033 
10. Koh YW, Lee SJ, Park SY. Prognostic significance of lactate dehydrogenase $\mathrm{B}$ according to histologic type of non-small-cell lung cancer and its association with serum lactate dehydrogenase. Pathol Res Pract. 2017;213:1134-1138. doi:10.1016/j.prp.2017.07.006

11. Gan J, Wang W, Yang Z, et al. Prognostic value of pretreatment serum lactate dehydrogenase level in pancreatic cancer patients: a meta-analysis of 18 observational studies. Medicine (Baltimore). 2018;97(46):e13151. doi:10.1097/MD.0000000000013151

12. Sonehara K, Tateishi K, Komatsu M, et al. Lung immune prognostic index as a prognostic factor in patients with small cell lung cancer. Thorac Cancer. 2020;11(6):1578-1586. doi:10.1111/17597714.13432

13. Luo Y, Yang Z, Li D, et al. LDHB and FABP4 are associated with progression and poor prognosis of pancreatic ductal adenocarcinomas. Appl Immunohistochem Mol Morphol. 2017;25:351-357. doi:10.1097/PAI.0000000000000306

14. Sun X, Sun Z, Zhu Z, et al. Expression of SIP1 is strongly correlated with LDHA and shows a significantly poor outcome in gastric cancer. Tumour Biol. 2015;36:7521-7530. doi:10.1007/s13277-015-3470-7

15. Girgis H, Masui O, White NM, et al. Lactate dehydrogenase A is a potential prognostic marker in clear cell renal cell carcinoma. Mol Cancer. 2014;13:101. doi:10.1186/1476-4598-13-101

16. Yu C, Hou L, Cui H, et al. LDHA upregulation independently predicts poor survival in lung adenocarcinoma, but not in lung squamous cell carcinoma. Future Oncol. 2018;14:2483-2492. doi:10.2217/fon2018-0177

17. Li SJ, Huang X, Xiao X, et al. Expression of LDHA in breast cancer and its clinical significance. J Chin Physician. 2017;19:207-209.

18. Kamisawa T, Wood LD, Itoi $\mathrm{T}$, et al. Pancreatic cancer. Lancet. 2016;388(10039):73-85. doi:10.1016/S0140-6736(16)00141-0

19. Ilic M, Ilic I. Epidemiology of pancreatic cancer. World J Gastroenterol. 2016;22:9694-9705. doi:10.3748/wjg.v22.i44.9694

20. Kim JE, Lee KT, Lee JK, et al. Clinical usefulness of carbohydrate antigen 19-9 as a screening test for pancreatic cancer in an asymptomatic population. J Gastroenterol Hepatol. 2004;19:182-186. doi:10.1111/j.1440-1746.2004.03219.x

21. Goonetilleke KS, Siriwardena AK. Systematic review of carbohydrate antigen (CA 19-9) as a biochemical marker in the diagnosis of pancreatic cancer. Eur J Surg Oncol. 2007;33:266-270. doi:10.1016/ j.ejso.2006.10.004
22. Chan A, Prassas I, Dimitromanolakis A, et al. Validation of biomarkers that complement CA19.9 in detecting early pancreatic cancer. Clin Cancer Res. 2014;20:5787-5795. doi:10.1158/1078-0432.CCR14-0289

23. Kaur S, Smith LM, Patel A, et al. A combination of MUC5AC and CA19-9 improves the diagnosis of pancreatic cancer: a multicenter study. Am J Gastroenterol. 2017;112(1):172-183. doi:10.1038/ ajg.2016.482

24. Balmaña M, Duran A, Gomes C, et al. Analysis of sialyl-Lewis x on MUC5AC and MUC1 mucins in pancreatic cancer tissues. Int J Biol Macromol. 2018;112(1):33-45. doi:10.1016/j.ijbiomac.2018.01.148

25. Capurso G, Signoretti M, Valente R, et al. Methods and outcomes of screening for pancreatic adenocarcinoma in high-risk individuals. World J Gastrointest Endosc. 2015;7:833-842. doi:10.4253/wjge.v7. i9.833

26. Le A, Cooper CR, Gouw AM, et al. Inhibition of lactate dehydrogenase a induces oxidative stress and inhibits tumor progression. Proc Natl Acad Sci USA. 2010;107(5):2037-2042. doi:10.1073/ pnas.0914433107

27. Jiang W, Zhou F, Li N, et al. FOXM1-LDHA signaling promoted gastric cancer glycolytic phenotype and progression. Int J Clin Exp Pathol. 2015;8:6756-6763.

28. Jelski W, Chrostek L, Szmitkowski M. The activity of class I, II, III, and IV of alcohol dehydrogenase isoenzymes and aldehyde dehydrogenase in pancreatic cancer. Pancreas. 2007;35(2):142-146. doi:10.1097/MPA.0b013e318053eae2

29. Jelski W, Zalewski B, Szmitkowski M. Alcohol dehydrogenase $(\mathrm{ADH})$ isoenzymes and aldehyde dehydrogenase (ALDH) activity in the sera of patients with pancreatic cancer. Dig Dis Sci. 2008;53 (8):2276-2280. doi:10.1007/s10620-007-0144-3

30. Jelski W, Kutylowska E, Laniewska-Dunaj M, et al. Alcohol dehydrogenase $(\mathrm{ADH})$ and aldehyde dehydrogenase (ALDH) as candidates for tumor markers in patients with pancreatic cancer. J Gastrointestin Liver Dis. 2011;20(3):255-259.

\section{Publish your work in this journal}

Cancer Management and Research is an international, peer-reviewed open access journal focusing on cancer research and the optimal use of preventative and integrated treatment interventions to achieve improved outcomes, enhanced survival and quality of life for the cancer patient.
The manuscript management system is completely online and includes a very quick and fair peer-review system, which is all easy to use. Visit http://www.dovepress.com/testimonials.php to read real quotes from published authors. 